\title{
BMJ
}

\section{Risk of colorectal cancer seven years after flexible sigmoidoscopy screening: randomised controlled trial}

\author{
Geir Hoff, professor, ${ }^{1,2}$ Tom Grotmol, professor , ${ }^{1}$ Eva Skovlund, professor , ${ }^{3}$ Michael Bretthauer, physician and \\ researcher, ${ }^{1,4}$ for the Norwegian Colorectal Cancer Prevention Study Groupfor the Norwegian Colorectal \\ Cancer Prevention Study Group
}

\begin{abstract}
${ }^{1}$ Norwegian Colorectal Cancer Prevention (NORCCAP) Centre, Cancer Registry of Norway, Montebello, NO-0310 Oslo, Norway

${ }^{2}$ Department of Medicine Telemark Hospital, NO-3710 Skien, Norway

${ }^{3}$ School of Pharmacy, University of Oslo, NO-0316 Oslo

${ }^{4}$ Department of Gastroenterology, Rikshospitalet University Hospital, NO-0027 Oslo

Correspondence to: G Hoff, Cance Registry of Norway, P O Box 5313 Majorstuen, 0304 Oslo, Norway hofg@online.no
\end{abstract}

Cite this as: BMJ 2009;338:b1846 doi:10.1136/bmj.b1846

\section{ABSTRACT}

Objective To determine the risk of colorectal cancer after screening with flexible sigmoidoscopy.

Design Randomised controlled trial.

Setting Population based screening in two areas in Norway-city of Oslo and Telemark county (urban and mixed urban and rural populations).

Participants 55736 men and women aged 55-64 years. Intervention Once only flexible sigmoidoscopy screening with or without a single round of faecal occult blood testing $(n=13823)$ compared with no screening ( $n=$ 41913).

Main outcome measures Planned end points were cumulative incidence and mortality of colorectal cancer after 5, 10, and 15 years. This first report from the study presents cumulative incidence after 7 years of follow-up and hazard ratio for mortality after 6 years.

Results No difference was found in the 7 year cumulative incidence of colorectal cancer between the screening and control groups (134.5 v 131.9 cases per 100000 person years). In intention to screen analysis, a trend towards reduced colorectal cancer mortality was found (hazard ratio $0.73,95 \%$ confidence interval 0.47 to $1.13, \mathrm{P}=0.16$ ). For attenders compared with controls, a statistically significant reduction in mortality was apparent for both total colorectal cancer (hazard ratio $0.41,0.21$ to 0.82 , $\mathrm{P}=0.011)$ and rectosigmoidal cancer $(0.24,0.08$ to 0.76 , $\mathrm{P}=0.016$ ).

Conclusions A reduction in incidence of colorectal cancer with flexible sigmoidoscopy screening could not be shown after 7 years' follow-up. Mortality from colorectal cancer was not significantly reduced in the screening group but seemed to be lower for attenders, with a reduction of $59 \%$ for any location of colorectal cancer and $76 \%$ for rectosigmoidal cancer in per protocol analysis, an analysis prone to selection bias.

Trial registration Clinical trials NCT00119912.

\section{INTRODUCTION}

More than 500000 estimated annual deaths from colorectal adenocarcinoma make this the third most common cause of deaths from cancer worldwide. Symptoms tend to appear late in the course of the disease, and early surgery remains the only option for cure. For the past decade, screening for colorectal cancer with flexible endoscopes has therefore been advocated in the United States. ${ }^{2}$ Several European countries have recently launched large scale colonoscopy screening programmes for the general population. ${ }^{34}$

Evidence shows that endoscopic screening may prevent colorectal cancer by detection and removal of premalignant, adenomatous polyps. ${ }^{5-7}$ This effect might, however, have been overestimated, as the extent of spontaneous regression of adenomas is largely unknown. ${ }^{8}$ Apart from a small scale trial on screening with flexible sigmoidoscopy, none of these studies investigating screening and polypectomy have been randomised trials. ${ }^{9}$ The World Health Organization recommends that screening programmes should be set up only when their effectiveness has been demonstrated. ${ }^{10}$ The United Kingdom National Screening Committee explicitly demands that this is based on randomised trials. ${ }^{11}$ The NORwegian Colorectal CAncer Prevention (NORCCAP) trial 1 is a population based randomised controlled trial for the prevention of colorectal cancer, comparing once only flexible sigmoidoscopy with no screening (usual care). The primary end point of the study is incidence of colorectal cancer, which was to be reported after 5,10 , and 15 years of follow-up.

This paper presents the first results from the NORCCAP trial 1 on the incidence of colorectal cancer after a minimum of six (range six to eight) years and mortality from colorectal cancer after a minimum of five (five to seven) years of follow-up. It is, to our knowledge, the first report to present data on the effect of screening with flexible sigmoidoscopy from a large scale population based randomised controlled trial.

\section{METHODS}

Population and participants

Norway has a unique person identification system, which uses a compulsory 11 digit identification number (including date of birth) for each person living in Norway; this secures registration of lifetime events and longitudinal data for epidemiological research. All residents aged 55-64 years living in the city of Oslo and Telemark County, Norway, who were registered 
and alive in the national population registry by November 1998 ( $\mathrm{n}=55736)$, were eligible for the NORCCAP trial 1. Of these, 13823 people (men and women, 1:1) were drawn by individual randomisation from the population registry and invited directly to once only flexible sigmoidoscopy screening (one step invitation procedure); this process mimicked invitation procedures for national screening programmes. ${ }^{12}$ An independent body (Statistics Norway) did the randomisations. Fifty per cent of those invited $(6908$ people, 1:1 randomisation within the screening group) were also asked to provide three consecutive stool samples on attendance for screening, to investigate the effect on compliance of adding a supplementary screening modality (faecal occult blood) with additional inconvenience for people screened. Screening for occult blood only was not an option.

People randomised to the control group ( $n=41913)$ were not offered any screening; they were not contacted, and follow-up was purely registry based. To obtain a wider age range for future decisions on screening policy in Norway, we decided towards the end of the study period to include an additional randomised sample of the population at age 50-54 years to be offered flexible sigmoidoscopy only or combined flexible sigmoidoscopy and faecal occult blood testing. Owing to short follow-up, data from this add-on study group will not be presented here.

\section{Screening intervention}

Screening examinations were done at two centres (one in each of the screening areas) between January 1999 and December 2000. Bowel preparation for flexible sigmoidoscopy was restricted to a $240 \mathrm{ml}$ sorbitol enema administered at the screening centre on attendance. Ordinary colonoscopes were used both for

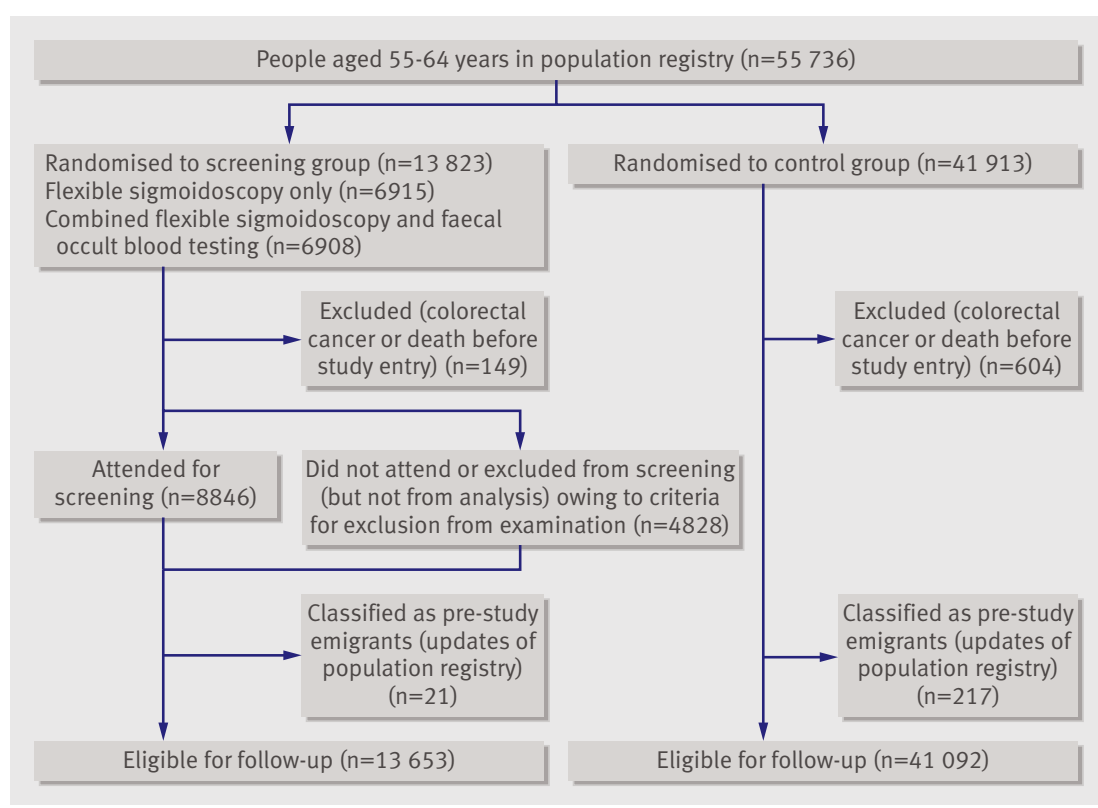

Fig 1| Flow chart for Norwegian Colorectal Cancer Prevention trial 1 cohort screened January 1999 to December 2001 flexible sigmoidoscopy screening and work-up colonoscopy for screen positives $(140 \mathrm{~cm}$ Olympus colonoscopes, Olympus Europa GmbH, Hamburg, Germany). The level reached was measured in centimetres from the anal verge with the endoscope in a straightened position, as judged by the endoscopist. A temporary satellite screening unit was set up for the inhabitants of the most peripheral parts of rural Telemark county, staffed and equipped by endoscopists and nurses from the main Telemark centre.

All lesions detected at the screening examinations were subjected to tissue sampling and histopathological diagnosis. For faecal occult blood testing, we used an immunochemical test (FlexSure OBT, BeckmanCoulter, Palo Alto, CA, USA).

We defined a positive screening test as any polyp $10 \mathrm{~mm}$ or more in diameter, any histologically verified adenoma irrespective of size, carcinoma, or a positive occult blood test. These findings qualified participants for full colonoscopy, which was also done at the screening centres. At colonoscopy, all polyps $4 \mathrm{~mm}$ or more in diameter were removed by diathermy loop resection, and hot or cold biopsy forceps were applied to smaller lesions. Twenty one per cent of people screened had colonoscopy. ${ }^{13}$ We defined screen detected colorectal cancers as lesions found at flexible sigmoidoscopy or during work-up colonoscopy of screen positive participants. After screening and treatment of screen positive participants, follow-up of individual participants has been restricted to usual public health care, which by and large implies no surveillance within the first five years. ${ }^{14}$

Before the start of the study, we established criteria for exclusion from the screening examination by personal contact with the people invited. People who fitted at least one of the criteria listed below were not screened but were included in the intention to screen analyses, as similar information was not available from the control group. The exclusion criteria were previous open colorectal surgery, need for long term attention and nursing services (somatic or psychosocial reasons, mental retardation), ongoing cytotoxic treatment or radiotherapy for malignant disease, severe chronic cardiac or pulmonary disease (New York Heart Association III-IV), lifelong anticoagulant treatment, admission to hospital for a coronary event during the previous three months, cerebrovascular accident during the previous three months, and residence abroad. Death or a diagnosis of colorectal cancer before the study entry date was available through national registries and was used as a criterion for exclusion from analyses.

\section{Study entry}

The date of entry into the study for the people in the screening group was the date of the screening appointment, evenly distributed throughout the two year screening period (January 1999 to December 2000) by central, random allocation. For data analyses, computerised random allocation of individual entry dates within the same two year time period was similarly 
done for all people in the control group. Thus, all people in the control group were assigned an individual study entry date. People who developed colorectal carcinoma or died before their individual study entry date were excluded from analyses in both groups.

\section{Outcomes}

The primary end point in the NORCCAP trial 1 is incidence of colorectal cancer to be reported after 5, 10 , and 15 years of follow-up on an intention to screen basis, in which all randomised, eligible people are included, regardless of their compliance with the screening examination. Further end points are mortality from and incidence of colorectal cancer within the reach of the flexible sigmoidoscope (rectum and sigmoid colon) for attenders (per protocol analysis).

\section{Follow-up}

We followed people in both groups through national registries. In Norway, reporting of data on any incident cancer to the cancer registry of Norway and on any cause of death to the Norwegian cause of death registry is compulsory. These registries are virtually $100 \%$ complete. ${ }^{15}$ We retrieved all incident cases of colorectal cancer in the study cohort from the main database at the cancer registry. The end of follow-up for incidence of colorectal cancer was 31 December 2006. The cancer registry of Norway uses a modified version of ICD7 (international classification of diseases, 7 th revision) for coding, in which "localised disease" constitutes Dukes' A and B and "advanced disease" constitutes tumours infiltrating neighbouring organs and Dukes' C. Information on cause specific death came in ICD-10 format from the Norwegian cause of death registry. As this registry has a one year delay compared with the cancer registry, end of follow-up for cause specific death in this paper is 31 December 2005.

This first report from the study came after a minimum of five years' follow-up on colorectal cancer mortality for all people included in the study. As the delay in registration of the cause of death is one year longer than the delay in registration of cancer incidence, this report comprises a minimum of six years' follow-up for incidence of colorectal cancer. Assessment of both the cause of death and colorectal cancer staging for the registries used was blinded to the group status of participants in the study.

\section{Sample size}

On the basis of data from the cancer registry of Norway, we expected an accumulated five year incidence of colorectal cancer of approximately 1\% (180 cases per 100000 person years) in the control group at age 60-69 years. Assuming 70\% compliance and 50\% coverage of the colorectal mucosa by flexible sigmoidoscopy, we regarded a 30\% reduction in incidence after five years in the intention to screen population as possible to achieve and definitely worth while to detect. With a 5\% significance level (two sided), we estimated the power to detect this difference to be $90 \%$ if 14000 people were invited for screening and 42000 people were allocated to the control group. This corresponds to approximately $75 \%$ power to detect a $25 \%$ reduction.

\section{Statistical methods}

We present results as cumulative incidence rates. In addition, we illustrate time to colorectal cancer by estimating the cumulative hazard function, which clearly shows non-proportionality. We analysed mortality from colorectal cancer and total mortality by using Cox proportional hazards model, as proportionality was not affected by screen detected colorectal cancers. We used the Nelson-Aalen method to estimate cumulative hazard rates in the screening and control groups. We censored all time to event data at the end of the follow-up period and at emigration. Additionally, for analyses of incidence of colorectal cancer and estimates of cumulative hazard we censored data at death and at diagnosis of colorectal malignancy other than adenocarcinoma.

\section{RESULTS}

Figure 1 shows the flow chart for the 55736 people who were randomised. Altogether, 753 cases of colorectal cancer or death occurred before the study entry date, including four people with previous colorectal cancer who were erroneously screened. This left 13653 people in the screening group and 41092 in the control group eligible for analyses (fig 1). Censoring owing to emigration occurred for 1196 people, and 21 people were censored as a result of colorectal malignancy other than colorectal carcinoma (13 neuroendocrine (carcinoid) tumours and eight squamous cell carcinomas). In the screening group, 459 people were excluded from examination, according to the

Accumulated cases (accumulated No/1000 people) of colorectal cancer in subsets of screening group and control group after six to eight years' follow-up

\begin{tabular}{|c|c|c|c|c|c|c|}
\hline & \multicolumn{5}{|c|}{ Screening group $(n=13653)$} & \multirow[b]{3}{*}{$\begin{array}{c}\text { Control group } \\
(n=41092)\end{array}$} \\
\hline & \multicolumn{3}{|c|}{ Attended $(n=8846)$} & \multirow[b]{2}{*}{$\begin{array}{c}{ }^{\star D i d} \text { not attend } \\
(n=4807)\end{array}$} & \multirow[b]{2}{*}{ Total } & \\
\hline & Screen detected & $\begin{array}{l}\text { Post-screen } \\
\text { detected }\end{array}$ & $\begin{array}{l}\text { Subtotal } \\
\text { attending }\end{array}$ & & & \\
\hline Localised colorectal cancer & 20 & 6 & 26 & 7 & $33(2.4)$ & $62(1.5)$ \\
\hline Advanced colorectal cancer & $11(1.2)$ & $29(3.3)$ & $40(4.5)$ & $38(7.9)$ & $78(5.7)$ & $262(6.4)$ \\
\hline Stage unspecified & 2 & 3 & 5 & 7 & $12(0.9)$ & $38(0.9)$ \\
\hline Total & 33 & 38 & $71(8.0)$ & $52(10.8)$ & $123(9.0)$ & $362(8.8)$ \\
\hline
\end{tabular}




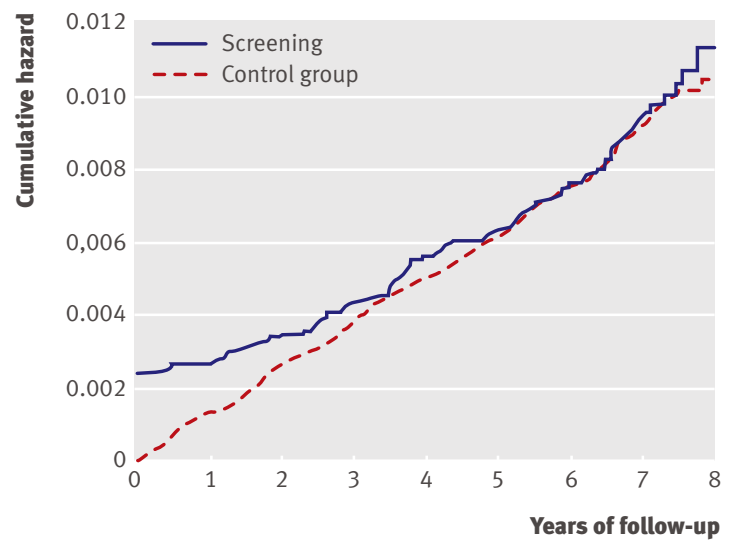

Fig 2 Cumulative hazard for colorectal cancer in screening and control groups

described exclusion criteria, but included in the intention to screen analyses.

The two groups were similar in the distribution of age (mean 59 years) and sex $(50 \%$ female in both groups). Of the 13653 people eligible, 8846 had a screening examination, giving an attendance rate of $64.8 \%$ (67\% with exclusion of those not examined owing to exclusion criteria). Compliance was slightly higher in women than in men $(65.9 \%(4554 / 6907) v$ $62.1 \%(4292 / 6916))$. The attending and non-attending groups had similar age distribution (mean 58.4 and 58 . 5 years, with a range of 55-64 for both groups). Mean insertion of the endoscope was $48.9(\mathrm{SD}=15.7) \mathrm{cm}$ for men and $44.0(14.2) \mathrm{cm}$ for women, as measured with a straightened endoscope. No severe complications occurred during flexible sigmoidoscopy. At screening, a neoplastic lesion was found in $19 \%(1685 / 8846)$ of people screened, and 5.0\% (440/8846) of attenders had high risk adenoma $(\geq 10 \mathrm{~mm}$ in diameter, high grade dysplasia or villous components) or invasive cancer. ${ }^{13}$ Out of 33 prevalent colorectal cancers detected by screening, 17 were in the 6915 people invited for flexible sigmoidoscopy only (2.5 per 1000 invited) and 16 in the 6908 people invited for combined flexible sigmoidoscopy and faecal occult blood testing (2.3 per 1000 invited).

The compliance for colonoscopy work-up was $97 \%$ (1812/1872). Ninety per cent (1617/1812) of colonoscopies took place without sedation or analgesia. The rate of caecal intubation at first attempt was $89 \%$ (1623/1812). In the 189 patients in whom baseline colonoscopy work-up was initially incomplete, the caecum was reached in 12 on a second attempt, double contrast barium enema was done in 52, and six were referred directly to surgery owing to distal tumour. Thus, 70 patients had their entire colon visualised during work-up or post-surgical surveillance. In the remaining 119 people with incomplete visualisation of the caecum, none had incident colorectal cancer at six to eight year follow-up. In addition to people with neoplasia at flexible sigmoidoscopy, colonoscopy work-up was done in 349 patients because of a positive faecal occult blood test $(\mathrm{n}=150)$, symptoms $(\mathrm{n}=65$, including one case of colorectal carcinoma), hyperplastic polyp $>10 \mathrm{~mm}(\mathrm{n}=28)$, poor bowel cleansing at flexible sigmoidoscopy $(\mathrm{n}=24)$, tissue sample lost at screening $(\mathrm{n}=22)$, and other reasons $(\mathrm{n}=60)$.

Median follow-up after inclusion in the trial was seven (range six to eight) years for incident colorectal cancer and six (range five to seven) years for mortality from colorectal cancer. The cumulative hazard rate starts at a high level in the screening group owing to detection of prevalent colorectal cancer cases at screening (fig 2). We found no difference in the cumulative hazard of colorectal cancer between the screening group and the control group (intention to screen analysis; $134.5 v 131.9$ cases per 100000 person years). The accumulated number of colorectal cancers after six to eight years of follow-up was 123 in the screening group, including 33 screen detected tumours, and 362 in the control group (table). In the two screening groups, 54 accumulated colorectal cancers occurred in the flexible sigmoidoscopy group (7.9 per 1000) and 69 (10.1 per 1000) in the group invited to combined flexible sigmoidoscopy and faecal occult blood testing. When we restricted the cumulative hazard plot to attenders and rectosigmoidal cancers only, the line crosses that of the control group, suggesting an effect of polypectomy for left sided colorectal cancer in those attending for screening (fig 3). The cumulative incidence of rectosigmoidal cancer was 35 cases in 8846 attenders (58 per 100000 person years) and 217 in 41092 controls (79 per 100000 person years) $(\mathrm{P}=0.103)$. Of 90 post-screen incident colorectal cancers in the screening group, 37 appeared among 6915 people invited for flexible sigmoidoscopy only (5.4 per 1000) compared with 53 in 6908 people invited for combined flexible sigmoidoscopy and faecal occult blood testing (7.7 per 1000).

A total of 24 of 13653 people in the screening group and 99 of 41092 in the control group died from colorectal cancer during follow-up. In the screening group as a whole (intention to screen), total mortality was reduced by $27 \%$ (hazard ratio $0.73,95 \%$ confidence interval 0.47 to $1.13, \mathrm{P}=0.16)$ for colorectal cancer and by $37 \%(0.63,0.34$ to $1.18, \mathrm{P}=0.15)$ for rectosigmoidal cancer compared with the control group.

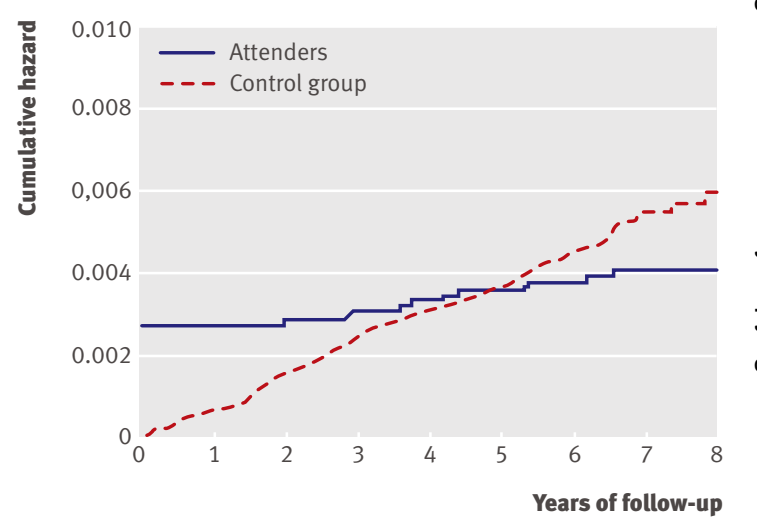

Fig 3 | Cumulative hazard for rectosigmoidal cancer among attenders compared with control group 
For those actually screened, total mortality was reduced by $59 \%$ (hazard ratio $0.41,0.21$ to 0.82 , $\mathrm{P}=0.011)$ for colorectal cancer and by $76 \%(0.24,0.08$ to $0.76, \mathrm{P}=0.016$ ) for rectosigmoidal cancer; this corresponded to three and 57 deaths from rectosigmoidal cancer. All cause mortality was similar in the screening group and the control group (hazard ratio 1.02, 0.98 to 1.07, $\mathrm{P}=0.28$ ).

We found a more favourable stage distribution for patients with screen detected colorectal cancer compared with those in the control group and the non-attending group (table). Almost half of the total number of colorectal cancers among attenders (33/71) were screen detected tumours, and 27 of the 38 post-screen colorectal cancers were localised proximal to the rectum and sigmoid colon. In attenders, 36 of 71 colorectal cancers were localised proximal to the rectosigmoid colon $(4.07 / 1000$ attenders) compared with 145 of 362 in the control group (3.53/1000 people, 3.74/1000 if nine tumours with undefined localisation were to be classified as proximal). Case fatalities from colorectal cancer diagnosed within the update period of cause of death registrations (that is, including 2005) were 9/66 (14\%) in the group of attenders $(2 / 33(6 \%)$ screen detected) compared with 15/38 (39\%) among non-attenders and $99 / 308(32 \%)$ in the control group. Excluding screen detected colorectal cancers, deaths from post-screen detected colorectal cancer among attenders ("interval cancers") were 7/33 (21\%).

\section{DISCUSSION}

This study showed a non-significant reduction in mortality of $27 \%$ from total colorectal cancer and $37 \%$ from rectosigmoidal cancer by intention to screen analysis and no reduction in accumulated incidence of colorectal cancer after seven years of follow-up. Among attenders, per protocol analysis (an analysis prone to selection bias) showed a significant 59\% reduction in incidence of total colorectal cancer and $76 \%$ reduced incidence of rectosigmoidoal cancer.

Reduction in mortality is the ultimate aim for any cancer screening programme. Endoscopic screening for colorectal neoplasia has the unique potential to go beyond that - to prevent colorectal cancer from developing by detection and removal of colorectal cancer precursor lesions (polypectomy of adenomas). Among several screening trials exploring this potential with flexible sigmoidoscopy, the Norwegian Colorectal Cancer Prevention trial 1 is the only trial with a one stage invitation design mimicking a national screening programme. $^{1216-18}$

\section{Is it too early to see an effect in intention to screen analyses?}

The flat incidence curve for rectosigmoidal cancer in attenders (fig 3) illustrates that attendance for flexible sigmoidoscopy screening is associated with a reduced risk of post-screening rectosigmoidal cancer, whether this is due to self selection of people at low risk choosing to attend, a high screening detection rate for established but asymptomatic cancers, or a genuine effect of polypectomy in preventing cancer. Attenders in the NORCCAP trial 1 were more often smokers but had a modestly lower risk profile, as judged by dietary habits and physical exercise, compared with the control group. ${ }^{19}$ Thus, a major "healthy screenee" effect does not seem to be occurring in this study.

We found a trend towards reduced mortality from colorectal cancer for both total colorectal cancer mortality (27\% reduction) and rectosigmoidal cancer mortality $(37 \%)$, but this was not statistically significant in intention to screen analysis, the single most important analysis from a public health perspective. Corresponding reductions in mortality among attenders were 59\% and $76 \%$, both statistically significant compared with the control group. When evaluating the latter result, one should bear in mind the inherent risk of selection bias in looking at attenders only.

Two main possibilities could explain the limited effect of screening with flexible sigmoidoscopy in this study: either the method is not effective in reducing incidence of colorectal cancer or the lag period for the development of cancer from precursor lesions is considerably longer than is commonly assumed. The second possibility is more likely, as Cuzick and associates pointed out. ${ }^{20}$ Contamination of the control group with colonoscopy is not a likely explanation for our findings, as no organised screening for colorectal cancer occurs in Norway and opportunistic screening is limited. According to an unpublished survey by $\mathrm{GH}$ in 2006, less than 5\% of colonoscopies in Norway are related to colorectal cancer screening activity compared with $50 \%$ in the United States. ${ }^{21}$

\section{How to evaluate with a high proportion of prevalent, screen detected cancers}

Prevalent (screen detected) colorectal cancers will dilute any incidence reducing effect of polypectomy. This effect has been estimated to last for two years after screening, ${ }^{22}$ but looking at results from the NORCCAP trial 1, it may last considerably longer than anticipated. Selectively excluding prevalent screen detected colorectal cancers from the analysis would give an apparently highly significant effect of screening with flexible sigmoidoscopy in reducing the incidence of rectosigmoidal cancer for people who attend, but this leads to severe bias. As the similar group of prevalent cancer cases cannot be identified and excluded from the control group, this type of analysis would overestimate the screening effect of flexible sigmoidoscopy. We have therefore chosen to base our conclusions on cumulative incidence. As flexible sigmoidoscopy screening showed no effect on total incidence of or mortality from colorectal cancer in the intention to screen analyses at this stage of followup, we did not do a separate analysis of the two screening modalities (flexible sigmoidoscopy and combined flexible sigmoidoscopy and faecal occult blood test). However, out of 13 cases of colorectal cancer subjected to both screening modalities, three were detected 


\section{WHAT IS ALREADY KNOWN ON THIS TOPIC}

Screening for colorectal cancer by endoscopy (flexible sigmoidoscopy and colonoscopy) has been advocated and implemented in several countries without previous randomised trials

Screening for faecal occult blood is a poor method for detection of colorectal cancer precursor lesions (adenomas) compared with endoscopy

Quantification of the effects of endoscopic screening has not been investigated through randomised trials

\section{WHAT THIS STUDY ADDS}

A non-significant reduction in mortality from colorectal cancer after six years of follow-up was seen in intention to screen analysis

The accumulated incidence rates of colorectal cancer were similar in the screening and the control groups, suggesting that seven years' follow-up may be too early to see any reduction in incidence

The risk of mortality from colorectal cancer for attenders was less than half that seen in controls; it was smaller for rectosigmoidal cancer than for all colorectal cancers

because of a positive faecal occult blood test when flexible sigmoidoscopy was negative.

Previous case-control and observational studies, as well as a small scale randomised trial, have indicated that endoscopic screening may reduce the incidence of colorectal cancer by 50-90\%.-79 Our results, with 33 screen detected cases of colorectal cancer and 38 cases of cancer diagnosed during the seven year postscreening period, indicate that screening with flexible sigmoidoscopy may detect close to $50 \%$ of neoplastic lesions already malignant or destined to turn malignant within a seven year timeframe. This is consistent with estimates from Danish and Canadian studies. ${ }^{2324}$ Some differences exist in diagnostic yield between ongoing studies of screening with flexible sigmoidoscopy. The pick-up rates for both any neoplasia (19\%) and advanced neoplasia $(5 \%)$ in our study were higher than or comparable to those in the ongoing British $(12 \%$ and $5 \%)$ and Italian $(12 \%$ and $3.4 \%)$ flexible sigmoidoscopy screening trials - all in 55-64 year age groups. ${ }^{131617}$ As we cannot yet foresee the outcome of a "once only" flexible sigmoidoscopy screening concept in terms of prevention of colorectal cancer, it will be very interesting to follow all these studies with their differences in baseline pick-up rates and polyp size dependent thresholds for a work-up colonoscopy. After all, most adenomas regress or do not develop into cancers, and new adenomas may appear as key players before the "once only" flexible sigmoidoscopy has had time to show an effect.

\section{"Interval cancers" fared no worse than cancer cases in controls}

The apparent reduction in deaths from colorectal cancer in people with screen detected cancers in the screening group $(6 \%$ colorectal cancer fatalities compared with $32 \%$ among controls) may be due to a more favourable stage distribution, but lead time bias is necessarily also an important factor that precludes formal comparison and valid conclusions. We were reassured to see that people with colorectal cancer that appeared after negative screening results fared no worse than those in the control group (21\% and 32\% of patients with colorectal cancer died from their cancer in the two groups). This suggests that length time bias (that is, the fast growing, aggressive tumours not being picked up by screening) may not be a problem in this study.

From a public health perspective, our results indicate that screening with flexible sigmoidoscopy may not reduce the overall incidence of colorectal cancer to the extent and within the timeframe expected at a population level, but it seems to be promising for reducing the incidence of rectosigmoidal cancer among attenders. The results also indicate a need to look into alternative screening modalities to be tested in randomised trials without waiting for further follow-up results of this trial or other ongoing trials on screening with flexible sigmoidoscopy. ${ }^{16-18}$ Although recommended for several years and launched as national screening programmes in several countries, screening with colonoscopy has not yet been subjected to adequately designed randomised trials. Our findings on polypectomy for prevention of colorectal cancer may not automatically be extrapolated from the rectosigmoidal segment and flexible sigmoidoscopy to the entire colon and colonoscopy, as the risk profiles and natural course may be quite different for proximal and distal colorectal cancers. The findings may, however, contribute to adequate design of randomised trials on screening with colonoscopy, including estimates of sample size and follow-up and sufficient awareness of the analytical problems caused by a high proportion of screen detected, prevalent colorectal cancers.

\section{Conclusions}

This seven year post-screening analysis indicates that the effect of screening with flexible sigmoidoscopy and polypectomy on reducing the incidence of colorectal cancer may be lower and will certainly occur later than anticipated. At this stage of follow-up, a large proportion of prevalent, screen detected colorectal cancers makes it uncertain whether the observed flattening of the incidence curve during the first years after screening is simply due to prevalent, screen detected colorectal cancers that would otherwise have appeared as incident colorectal cancers or a genuine preventive effect on colorectal cancer by removal of adenomas. The findings, however, suggest an incidence reducing effect on distal colorectal cancer, matched by a $76 \%$ reduction in mortality for people attending screening.

We thank Mette Kalager, Bjarte Aagnes, Odd Aalen, Steinar Tretli, Tor Haldorsen, and Freddie Bray for valuable discussions, as well as the Norwegian Colorectal Cancer Prevention (NORCCAP) trial staff for enthusiastic assistance

The NORCCAP Study Group comprises Michael Bretthauer, Erik Carlsen, Tor Jac Eide, Tom Grotmol, Geir Hoff, Tor Iversen, Stener Kvinnsland, 
Frøydis Langmark, Jon Lekven, Jørn Schneede, Eva Skovlund, Kjell Magne Tveit, and Morten Vatn.

Further collaborators were Knut Bjerke, Per Efskind, Ghous Gondal, Kai Brynjar Hagen, Gert Huppertz-Hauss, Yngve Haugstvedt, Bjørn Hofstad, Solveig Thorp Holmsen, Anita Jørgensen, Inger Kristin Larsen, Per G Lund-Larsen, Idar Lygren, Volker Moritz, Sverre Nyhus, Odd Søreide, Espen Thiis-Evensen, Lisbeth Tranebjærg, Aage Tverdal, Per Wium, and Lars Ødegård.

The NORCCAP external advisory committee comprises Hans-Olov Adami, Sandra J Lee (both Harvard School of Public Health, Boston, USA), and Douglas K Rex (Indiana University School of Medicine, Indianapolis, USA) Contributors: GH, TG, and ES planned the study together with members of the NORCCAP Study Group. Together with MB, they drafted and refined the manuscript. All four authors also contributed to the statistical analysis with full access to all data. All authors wrote and approved the final draft of the manuscript. Frøydis Langmark (director, Cancer Registry of Norway, Oslo) is the guarantor.

Funding: NORCCAP trial 1 was funded by grants from the Norwegian Cancer Society and the Norwegian Ministry of Health. The authors are independent from the funders of the NORCCAP trial 1.

Competing interests: None declared.

Ethical approval: The regional committee for medical research ethics and the Norwegian Data Inspectorate approved the NORCCAP trial 1 protocol. All participants in the screening group have been informed about the nature and purpose of the study, and all those who attended screening provided written informed consent in advance.

1 Parkin DM, Bray F, Ferlay J, Pisani P. Global cancer statistics, 2002. CA Cancer J Clin 2005;55:74-108.

2 Rex DK, Johnson DA, Lieberman DA, Burt RW, Sonnenberg A Colorectal cancer prevention 2000: screening recommendations of the American College of Gastroenterology. Am J Gastroenterol 2000;95:868-77.

3 Regula J, Rupinski M, Kraszewska E, Polkowski M, Pachlewski J, Orlowska J, et al. Colonoscopy in colorectal-cancer screening for detection of advanced neoplasia. N Engl J Med 2006;355:1863-72.

4 Rozen P, Winawer SJ. Report of the OMED colorectal cancer screening committee meeting, New Orleans, 2004-in collaboration with the IDCA. Eur J Cancer Prev 2004;13:461-4.

5 Winawer SJ, Zauber AG, Ho MN, O’Brian MJ, Gottlieb LS, Sternberg SS, et al. Prevention of colorectal cancer by colonoscopic polypectomy. N Engl J Med 1993;329:1977-81.

6 Lieberman DA, Weiss DG. One-time screening for colorectal cancer with combined faecal occult blood testing and examination of the distal colon. N Engl J Med 2001;345:555-60.

7 Schoenfeld P, Cash B, Flood A, Dobhan R, Eastone J, Coyle W, et al. Colonoscopic screening of average-risk women for colorectal neoplasia. N Engl J Med 2005;352:2061-8.

8 Loeve F, Boer R, Zauber AG, van Ballegooijen M, van Ootmarssen G], Winawer SJ, et al. National polyp study data: evidence for regression of adenomas. Int J Cancer 2004;111:633-9.

9 Thiis-Evensen E, Hoff GS, Sauar J, Langmark F, Majak BM, Vatn MH. Population based surveillance by colonoscopy: effect on the incidence of colorectal cancer. Scand J Gastroenterol 1999;34:414-20.

10 Wilson JMG, Jungner G. Principles of practice of screening for disease Geneva: World Health Organization, 1968.

11 UK National Screening Committee. Criteria for appraising the viability, effectiveness and appropriateness of a screening programme. www.screening.nhs.uk/criteria.

12 Bretthauer M, Gondal G, Larsen IK, Carlsen E, Eide TJ, Grotmol T, et al. Design, organization and management of a controlled population screening study for detection of colorectal neoplasia. Scand Gastroenterol 2002;37:568-73.

13 Gondal G, Grotmol T, Hofstad B, Bretthauer M, Eide TJ, Hoff G. The Norwegian colorectal cancer prevention (NORCCAP) screening study: baseline findings and implementations for clinical work-up in age groups 50-64 years. Scand J Gastroenterol 2003;38:635-42.

14 Hoff G, Sauar J, Hofstad B, Vatn MH. The national guidelines for surveillance after polypectomy: 10-year intervals. Scand J Gastroenterol 1996;31:834-6.

15 Cancer in Norway 2005. Oslo: Cancer Registry of Norway, 2006.

16 UK Flexible Sigmoidoscopy Screening Trial Investigators. Single flexible sigmoidoscopy screening to prevent colorectal cancer: baseline findings of a UK multicentre randomized trial. Lancet 2002;359:1291-300.

17 Segnan N, Senore C, Andreoni B, Aste H, Bonelli L, Crosta C, et al. Baseline findings of the Italian multicenter randomized controlled trial of "once-only sigmoidoscopy"-SCORE. J Natl Cancer Inst 2002;94:1763-72.

18 Palitz AM, Selby JV, Grossman S, Finkler LJ, Bevc M, Kehr C, et al. The colon cancer prevention program (CoCaP): rationale implementation, and preliminary results. HMO Pract 1997;11:5-12.

19 Larsen IK, Grotmol T, Almendingen K, Hoff G. Lifestyle characteristic among participants in a Norwegian colorectal cancer screening trial. Eur J Cancer Prev 2006;15:10-9.

20 Cuzick J, Cafferty FH, Edwards R, Møller H, Duffy SW. Surrogate endpoints for cancer screening trials: general principles and an illustration using the UK Flexible Sigmoidoscopy Screening Trial. Med Screen 2007;14:178-85.

21 Seeff LC, Richards TB, Shapiro JA, Nadel MR, Manninen DL, Given LS, et al. How many endoscopies are performed for colorectal cancer screening? Results from CDC's survey of endoscopic capacity. Gastroenterology 2004;127:1670-7.

22 Atkin WS, Edwards R, Wardle J, Northover JMA, Sutton S, Hart AR, et al. Design of a multicentre randomised trial to evaluate flexible sigmoidoscopy in colorectal cancer screening. J Med Screen 2001;8:137-44.

23 Rasmussen M, Kronborg O, Fenger C, Jørgensen OD. Possible advantages and drawbacks of adding flexible sigmoidoscopy to Hemoccult-II in screening for colorectal cancer: a randomized study. Scand J Gastroenterol 1999;34:73-8

24 Singh H, Turner D, Xue L, Targownik LE, Bernstein CN. Risk of developing colorectal cancer following a negative colonoscopy examination: evidence for a 10-year interval between colonoscopies. JAMA 2006;295:2366-73.

Accepted: 8 April 2009 